\title{
Estimating Survival Probability of Drug Users with Application to Drug and Substance Abuse in Kenya
}

\author{
Robert Kasisi, Joseph Koske, Mathew Kosgei \\ Department of Mathematics and Computer Science Moi University, Eldoret, Kenya \\ Email address: \\ rkasisi@gmail.com (R. Kasisi), mkkosgei@yahoo.com (J. Koske), koske4@yahoo.co.uk (M. Kosgei) \\ To cite this article: \\ Robert Kasisi, Joseph Koske, Mathew Kosgei. Estimating Survival Probability of Drug Users with Application to Drug and Substance Abuse \\ in Kenya. American Journal of Theoretical and Applied Statistics. Vol. 6, No. 6, 2017, pp. 284-289. doi: 10.11648/j.ajtas.20170606.14
}

Received: June 28, 2017; Accepted: July 17, 2017; Published: November 27, 2017

\begin{abstract}
The contemporary studies on drug abuse have blamed the increasing menace of drug abuse on failure of governments to enact adequate laws prohibiting drug abuse and failure to place strict border controls to prevent entry of drugs. Others have blamed social media and modernization as key players towards the current trends of drug abuse. As a result studies have shifted from studying factors leading to drug abuse as these seem to be obvious to studying covariates that leading to improved probabilities of recovery upon treatment. Female substance users are said to be proportionately more likely to recover from drug use than male substance abusers. However studies have showed that female drug users experience low turnout for treatment from drug abuse. With the increasing trend of women drug users seeking treatment there is an urgent need to estimate survival probability of drug use subjects based on marital status, age, gender and job status. This study sought to determine the survival probability of drug users in Kenya for the period between July 2013 and June 2015. Kaplan Meier analysis was used to determine the survival probability of a subject entering into drug use at different stages of life based on predictive covariates. Survival probability of drug users based on age, gender, marital status and employment status was determined. The study recommended that there significant differences in survival probability based on gender, age, marital status and employment status. Therefore the study recommended that treatment services be tailored on treating subjects based on these predictive covariates.
\end{abstract}

Keywords: Survival Probability, Kaplan Meier Analysis, Intervention Programs, Hazard Rate, Drug Abuse

\section{Introduction}

Illicit drug use is a serious health problem to many countries. In Kenya drug abuse has continued to raise attention due to the increasing population of drug users. Some of the abused drugs include heroin, cocaine, tobacco and cannabis. However substances such as tobacco are not prohibited above certain age limit, use of drugs such as cocaine, heroin and cannabis is prohibited.

Drug abuse has adverse health effects (Degenhardt and Hall (2012). These effects include the acute toxic effect, the acute effect of intoxication (such as Accidental injury and violence related to alcohol intake), development of dependence and adverse health effects of continued regular use (for example chronic somatic disease and mental disorders). Rehm et al., (2009) states that alcohol use related disorders are the most significant disease categories for the global burden of disease. Research has also showed that five percentage of all deaths in young people between 15-29 years worldwide can be related to alcohol use. The majority of those alcohol-related deaths are linked to accidents (Gore et al., 2011; Rehm et al., 2009). Potential adverse health effects of substance use are more often related to acute toxic effect and acute effect of intoxication (such as accidental injury and violence related to alcohol intake) in adolescents than when compared to adults. Relatively few adverse health effects from dependence and continued regular use (such as chronic somatic disease) will be manifested during the adolescent years. However, regular use of substances has shown to have a major impact on future mental well-being, which in turn may be linked to different types of negative health outcomes (Volkow \& Li, 2005).

Many subjects enter into drug abuse due different reasons. A study conducted by (Beckman 1978) found that many women have low self-esteem over men especially when faced with challenges. This may lead them to drug abuse as a form 
of consolation. Depression is another common factor that leads to women entering into drug abuse (e.g. Culbertson 1997, Kesller et al, 1994; Weissman and Klerman 1997; Weissman and Paykel 1974). The ratios of Female to male drug abuser as shown in past studies are in the range of about 2:1 to $4: 1$ and statistical evidence suggests that gender difference is more likely to exist in more developed cultural settings (Culbertson 1997).

Carroll et al (1994a) states that interventions targeted at relapse prevention have been tested for efficacy studies. Interestingly these studies have not shown full relapse prevention. However relapse prevention treatment tend to indicate that benefit is achieved and also that substantial room exist for better efficacy. In a study of cocaine users, Carroll et al (1991) found that $57 \%$ attained three week or more of continuous abstinence and $43 \%$ met a criterion of recovery at the end of a twelve week treatment program In a later study, Carroll et al (1994b) also found some evidence that gains attained during acute treatment were maintained in a one year follow up of relapse prevention and pharmacotherapy for cocaine abuse. Treatment gains were maintained and even increased to some extent for the relapse prevention-alone condition. Gender differences in the efficacy of relapse prevention treatments have not been reported (Karla Moras, 2014). A 2012 study published in psychiatry research found that 100 women who received inpatient or day treatment for anorexia nervosa, $41 \%$ relapsed within one year. A study on relapse rates in bulimics assessed subjects before and after treatment and then again after 2 years. Within 2 years, $31 \%$ had relapsed with the majority of relapses occurring within the first 6 months.

A study done by Deleon (1993) found that there were no significant differences in survival rates based on gender. However a study by (Saunders et al 1993) found that there was a significant difference in the rate of survival based on gender. However from these past studies different authors give conflicting findings on significant covariates based on their geographical location. Therefore it is inconclusive to determine the significant covariates and determine survival probabilities of drug use subjects based on studies from different geographical locations. This study is motivated by lack of conclusive findings of previous studies and was motivated to determine survival probabilities of drug use in Kenya.

\section{Method}

\subsection{Survival Function}

Survivor function is the probability that the survival time of a randomly selected subject is greater than or equal to the specified time. It gives the probability of a subject surviving beyond a specified time. The distribution of survival time has the attribute of three functions namely the survivorship function, probability density function, and the hazard function. If we let $\mathrm{T}$ to be a random variable associated with the survival times, $t$ is the specified value of the random variable T. Then $f(t)$ is the underlying probability density function of the survival time T. $F(t)$, is the cumulative distribution function and it represents the probability that an individual selected at random will have a survival time less than $\mathrm{t}$, some stated value, which is given as

$$
F(t)=p(T<t)=\int_{0}^{t} f(x) d x, t \geq 0
$$

From the above, the survivor function, $S(t)$ may be written as

$$
S(t)=P(T \geq t)=1-F(t), t \geq 0
$$

From equations 2.1 .1 and 2.1 .2 the relationship between $f$ (t) and $\mathrm{S}(\mathrm{t})$ can be derived as

$$
f(t)=\frac{d}{d t} F(t)=\frac{d}{d t}(1-s(t))=\frac{-d}{d t} s(t), t \geq 0
$$

$t$ ranges from 0 to infinity. Survivor functions have the characteristics that:

\subsection{Comparison of Survival Functions}

We first obtain statistics which provide a description of the overall survival experience, the survival and hazard functions, then proceed with a comparison of the survival experience of data subgroups. These subgroups may be defined by the covariate values which are deemed to be related to survival times. When comparing groups of subjects, one should begin with a graphical display of the data in each group.

In studies of survival time, we graph the Kaplan-Meier estimator of the survival function for each of the subgroups. That is, plotting the corresponding estimates of the two survivor functions on the same axes of Kaplan Meier estimator. Generally if the plot shows that the pattern of one survival function lies above another, it would mean the group defined by the upper curve lived longer, or had a more favorable survival experience than the group defined by the lower curve. However, the statistical question is whether the observed difference seen on the plot is significant. This can be answered using an appropriate statistical test (Hosmer et $a l, 1999)$. The general form of test statistic that deal with this issue is given as

$$
\begin{aligned}
Q & =\left[\frac{\sum_{i=1}^{m} w_{i}\left(f_{1 i}-\hat{e}_{1 i}\right)}{\sum_{i=1}^{m} w_{i}{ }^{2} \hat{v}_{1 i}}\right] \\
\hat{e}_{1 i} & =\frac{n_{1 i} f_{i}}{n_{i}} \text { and } \hat{v}_{1 i}=\left[\frac{n_{o f} n_{1 i} f_{i}\left(f_{1 i}-\hat{e}_{1 i}\right)}{n_{i}{ }^{2}\left(n_{i}-1\right)}\right]
\end{aligned}
$$

In the above equation

$m$ is the number of rank-ordered failure (relapse) times, $n_{o i}$ is the number of individuals at risk at observed survival time $t_{(i)}$ in group 0 (male group), $n_{i 1}$ is the number of individuals at risk at observed survival time $t_{(i)}$ in group 1 (female group), $f_{0 i}$ is the number of observed relapses in group 0 , $f_{i 1}$ is the number of observed relapses in group $1, n_{i}$ is the total number of individuals or risk prior to time $t_{(i)}, f_{i}$ is the total number of relapses at time $t_{(i)}$ and $w_{i}$ is the weight for 
censor adjustment at relapse time $t_{(i)}$.

The contribution to the test statistic depends on which of the various tests is used, and each can be expressed in form of a ratio of weighted sums over the observed times of survival. Under the null hypothesis that the two survival functions are the same and while assuming that the censoring experience is independent of the subgroup, and that the total number of observed events and the sum of the expected number of events is significantly large, $Q$ follows a chisquare distribution with one degree of freedom. We can also use the test based on $\mathrm{Q}$ above to compare more than two subgroups. The log rank test is a special case of Q used in this study.

\subsection{Log Rank Test}

The log rank test, also called the Cox-Mantel test, is the most widely used test statistic. Log rank test statistic is based on weights equal to one, i.e $w_{i}=1$. Therefore, the log rank test statistic becomes

$$
Q_{G w t}=\frac{\left[\sum_{i=1}^{m}\left(f_{1 i}-\hat{e}_{1 i}\right)\right]^{2}}{\sum_{i=1}^{m} \hat{v}_{1 i}}
$$

\section{Results}

\subsection{Difference in Gender Survival Rate}

To determine if there were gender differences in the rate of survival from drug abuse based on gender, log rank test was conducted. Results showed a log rank test of $=47.67$ on 1 degrees of freedom $p$ value $=5.209 \times 10^{-12}<0.05$ which was significant. Therefore it was concluded that indeed there existed significant differences in the rate of survival among male and female subjects.

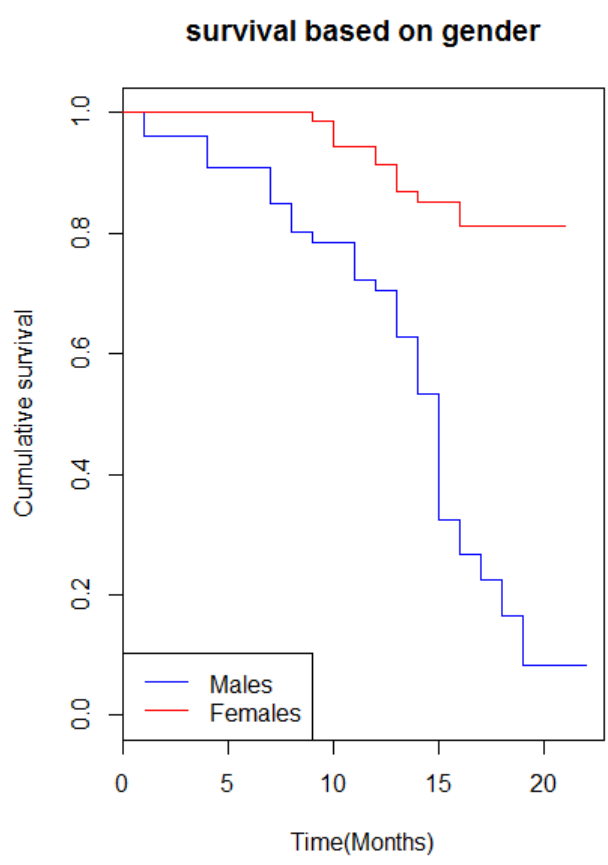

Figure 1. Survival probability based on gender.
Table 1. Log rank test for difference in gender survival rate.

\begin{tabular}{llllll}
\hline & $\mathbf{N}$ & Observed & Expected & $\frac{(\boldsymbol{O}-\boldsymbol{E})^{2}}{\boldsymbol{E}}$ & $\frac{(\boldsymbol{O}-\boldsymbol{E})^{2}}{\boldsymbol{V}}$ \\
\hline gender $=1$ & 76 & 51 & 24.5 & 28.7 & 50.5 \\
gender=2 & 86 & 12 & 38.5 & 18.3 & 50.5 \\
\hline
\end{tabular}

$\chi^{2}=35.67$ on 1 degrees of freedom $\mathrm{p}$ value $=2.341 \times 10^{-9}<0.05$

Female subjects had higher survival rate (61.14\%) compared to their male counterparts (32.21\%) [Figure 1]. This was consistent with previous studies (Wills, Yaeger, \& Sandy, 2003; Pitel et al., 2012). The study also found that females were more likely to quit drug use compared to their male counterparts.

\subsection{Survival Probability Based on Employment Status}

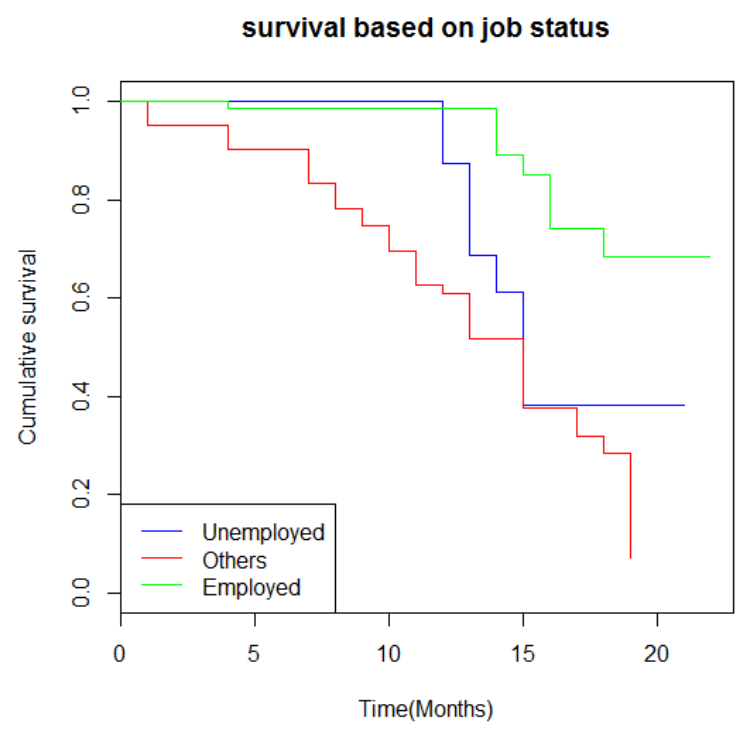

Figure 2. Survival probability based on employment status.

Results of table 2 showed that there were significance differences in survival rate among Employed, unemployed and those who specified any other category. This was supported by a Wald statistic of 33.9 which corresponded to a $\mathrm{p}$ value of $4.42 \times 10^{-8}<0.05$ and therefore was significant. Figure 2 also showed that employed subjects had higher survival rate compared to the other categories.

Table 2. Log rank test for employment status.

\begin{tabular}{|c|c|c|c|c|c|}
\hline & $\mathbf{N}$ & Observed & Expected & $\frac{(O-E)^{2}}{E}$ & $\frac{(\boldsymbol{O}-E)^{2}}{V}$ \\
\hline Job status $=1$ & 17 & 9 & 7.34 & 0.376 & 0.452 \\
\hline Job status $=2$ & 62 & 39 & 19.73 & 18.813 & 29.389 \\
\hline Job status $=3$ & 83 & 15 & 35.93 & 12.190 & 30.679 \\
\hline
\end{tabular}

Chisq $=33.9$ on 2 degrees of freedom, $\mathrm{p}=4.42 \mathrm{e}-08$.

\subsection{Survival Probability of Drug Users Based on Marital Status}

Information collected from medical records of each patient on marital status was carried out in order to study its relationship of study covariates with survival rate. The study population was divided into three groups based on marital history: married, unmarried and divorced. Kaplan-Meier 
method was used to determine the observed cumulative survival probability over time by calculating the proportion surviving due to drug use. The prevalence of unmarried individuals was higher compared to the married individuals. The study found that married individuals had higher survival rate compared to the rest of the categories [figure 3]. Married individuals had a survival rate of $67.89 \%$, divorced or separated individuals had a survival rate of $48.86 \%$ while the single individuals had a survival rate of $29.15 \%$. Marital status is also an important factor in helping reduce use of drugs. In most cases married individuals tend to be under pressure from their spouses to quit use of drugs and the need to care of the family also pushes the individuals to consider quitting drug abuse. This is in agreement with Ndetei, 2009 and NACADA 2007 who found that marriage reduces the risk of drug abuse. A study by Merete (2005) also showed that children born from mothers who are not married are at a higher risk of drug abuse $(75.6 \%)$ followed by single parents $(19.5 \%)$ and the least are those who are married.

Results of table 3 showed that there significance differences in survival rate among single, married, and divorced subjects. This was supported by a Wald statistic of 9.4 which corresponded to a $\mathrm{p}$ value of $0.00926<0.05$ and therefore was significant.

\subsection{Log Rank Test for Marital Status}

Table 3. Log rank test for marital status.

\begin{tabular}{llllll}
\hline & N & Observed & Expected & $\frac{(\boldsymbol{O}-\boldsymbol{E})^{2}}{\boldsymbol{E}}$ & $\frac{(\boldsymbol{O}-\boldsymbol{E})^{\mathbf{2}}}{\boldsymbol{V}}$ \\
\hline $\begin{array}{l}\text { Marital } \\
\text { status=1 }\end{array}$ & 39 & 8 & 17.2 & $4.96 \mathrm{e}+00$ & $7.29 \mathrm{e}+00$ \\
$\begin{array}{l}\text { Marital } \\
\text { status=2 }\end{array}$ & 61 & 23 & 23.0 & $4.34 \mathrm{e}-05$ & $7.34 \mathrm{e}-05$ \\
$\begin{array}{l}\text { Marital } \\
\text { status=3 }\end{array}$ & 62 & 32 & 22.8 & $3.73 \mathrm{e}+00$ & $6.35 \mathrm{e}+00$ \\
\hline
\end{tabular}

Chisq $=9.4$ on 2 degrees of freedom, $\mathrm{p}=0.00926$.

survival based on marrital status

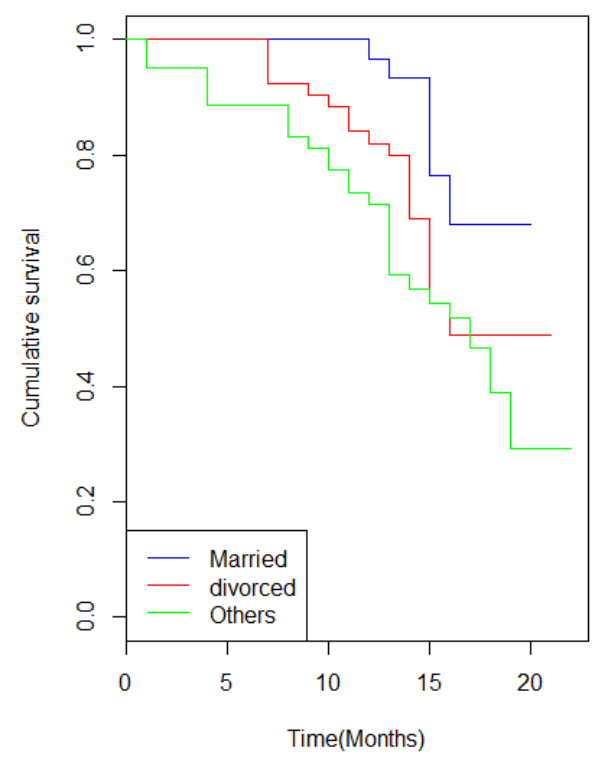

Figure 3. Survival probability based on marital status.

\subsection{Survival Rate Based on Age}

Survival based on type of Age

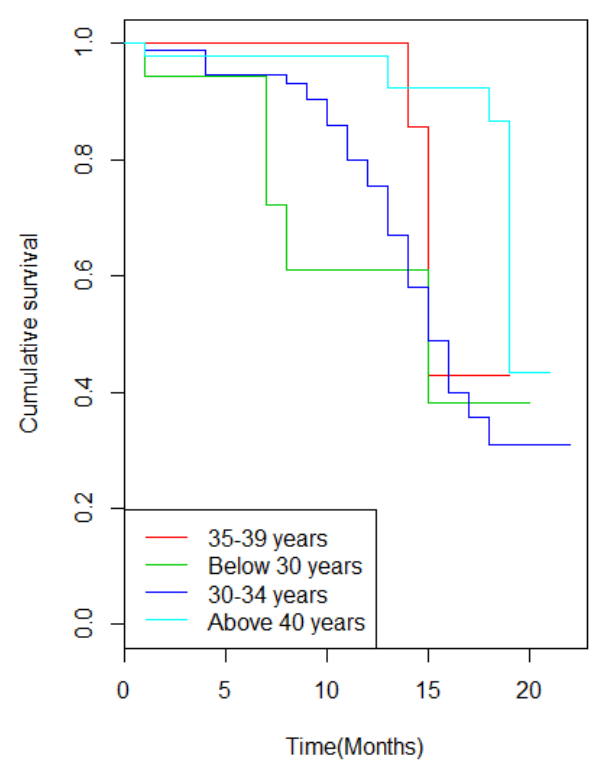

Figure 4. Survival probability based on age.

Subjects aged above 40 years had a survival rate of $43.33 \%$, those aged between $35-39$ years had a survival rate of $42.86 \%$, followed by $30-34$ years with a survival rate of $38.92 \%$. This was then followed by those aged below 30 years who had the least survival times of $30.92 \%$. This could be attributed to the fact that youth are highly exposed to many factors that contribute to drug abuse. These factors include peer pressure, stress and unemployment. Subjects aged above 30 years usually have higher survival rate due to the fact that they are under pressure from their spouses and employers to quit drug use [figure 4]. The findings are in agreement with other researchers whose findings have shown that age is significant in influencing drug abuse (Sutherland, 2008 \& arteaga, et al. 2010)

\section{Discussion}

After careful analysis the study obtained an overall survival rate of $36.9 \%$. This was similar to the relapse rates summarized by Greenwood et al. and Ramo and Brown, and Brown et al. Therefore, it is necessary to monitor and supervise the addicts' treatment to reduce the relapse rate, which should be implemented more effectively and accompanied with the contribution of addicts' families. Kaplan-Meier method was used to determine the observed cumulative survival probability over time due to drug use. The prevalence of drug use among unemployed subjects was higher compared to the employed individuals.

There were noteworthy differences in the survival time between the married, single and widowed or divorced subjects, which were 15.222 (CI 95\%: 13.523-16.92), 18.312 (CI 95\%: 17.323-19.301) and 16.768 (CI 95\%: 15.437-18.1) months, respectively. Mukherjee argued that lower relapse 
rate in married people rather than singles and divorced or separated persons can be due to family support and financial security, which are critical for recovery and social rehabilitation. In addition, in the study of Hosseini, marital duration played a significant role in survival time.

Employed individuals had a survival rate of $68.46 \%$, unemployed individuals had a survival rate of $38.19 \%$ while the rest of the subjects had a survival rate of $7.08 \%$. The impact of job status on survival rate has been assessed by several studies indicating that employment is associated with higher survival rate from drug use. Richardson believed that employment is usually upheld as a main consequence, indicator of the context of drug abuse treatment and recovery. This can be attributed to enabling the subject to attend treatment for drug abuse. The subject can be able to afford a higher medical care. Also the risk of losing a job due to drug abuse may also push the subjects to attend medical care (Bauld et al., 2010.). However a few studies have associated employment to increased drug abuse as the individuals can now afford to pay for the drugs of their choice. Noteworthy differences in the survival time and survival probability were also obtained for age and gender of the subjects.

\section{Conclusion}

From the study results, it was observed that female drug users had higher survival rate compared to the male drug users. The study reveals that youth are at higher risk of indulging into drug abuse compared to the older population. It is therefore important to have more intensive treatment programs for younger subjects compared to the older ones. Those subjects who had historical backgrounds of drug use were more exposed to drug use and therefore it is recommended that they attend inpatient treatment programs to improve their recovery rates. It was also observed that married people had a higher survival rates compared to the unmarried and divorced subjects and observed that injecting drug users were more likely to quit drug use compared to oral drug users. Therefore, these results provide a foundation of evidence and an essential element for raising public awareness, advocacy and improving health care service delivery with regards to drug abuse. Continued monitoring and evaluation of the drug use survival estimates is a vital component to developing future targeted and effective programs and policies in Kenya.

The study recommends setting up rehabilitation facilities within reach for the subjects to enable early detection and treatment of drug abuse and anti-drug abuse campaigns should also be targeted to fight multiple drug use.

\section{References}

[1] Barnett, E., et al. (2012). Motivational Interviewing for adolescent substance use: A review of the literature. Addictive Behaviors, 37 (12), 1325-1334.

[2] Bauld, L., Hay, G., McKell, J. and Carroll, C. 2010 "Problem drug users' experiences of employment and the benefit system” Department for Work and Pensions, 120: 45 -86.

[3] Berggren, U., Berglund, K., Fahlke, C., Aronsson, E., Eriksson, (2011). Attitudes toward the integration of smoking cessation treatment into drug abuse clinics. Journal of psychoactive drugs.

[4] Carroll, (1994a). Treatment for Stimulant Use Disorders, NIDA, American Journal of Psychiatry.

[5] Carroll, (1994b). A Cognitive-Behavioral Approach: Treating Cocaine Addiction, NIDA, American Journal of Psychiatry.

[6] Carroll, K, Rounsaville, B, Keller, D. Relapse prevention strategies for the treatment of cocaine abuse. American Journal of Drug and Alcohol Abuse. 1991; 17:249-265.

[7] Karla moras, (2014). The relationship between cocaine craving, psychosocial treatment, and subsequent cocaine use American Journal of Psychiatry.

[8] Culbertson, F. M. (1997). Depression and Gender: An International Review. American Psychologist.

[9] De Leo, J. A., \& Wulfert, E, (2013). Problematic internet use and other risky behaviors in college students: An application of problem-behavior theory. Psychology of Addictive Behaviors, 27 (1), 133-141.

[10] Fisher \& Roget. N, (2006). The Drug Abuse Treatment Outcomes. Journal of psychoactive drugs.

[11] Gore F. M, Bloem P. J. N, Palton G. C. (2011). Global burden of disease in young people aged 10-24 years: A systematic analysis. Lancet, 2011 vol 377.

[12] Hosmer, D. W. and Lemeshow, S. (1999). Applied Survival Analysis: Regression Modeling of Time to Event Data. Wiley, New York.

[13] Kaplan E. L and Paul Meier, (1958). Nonparametric Estimation from Incomplete Observations. Journal of the American Statistical Association, Vol. 53, No. 282.

[14] Hosmer D. W., and Lemeshow S., (2000). Applied Logistic Regression. Second Edition, John Wiley \& Sons.

[15] Greenwood G. L, Woods W. J, Guydish J, Bein E. (2001). Relapse outcomes in a randomized trial of residential and day drug abuse treatment. Journal of Substance Abuse Treatment, 20 (1):15-23.

[16] Kessler, (1994). Women and depression: Risk factors and treatment issues. American psychological association.

[17] kumpter, (2007). Substance abuse cycle intervention and prevention for children of substance abusers. Archives of General Psychiatry, 49, 716-722.

[18] Marsh,jeane C. Cao, Dingcai D’Aunno,Thomas, (2004). Gender differences in the impact of comprehensive services in substance abuse treatment. Journal of Substance Abuse Treatment 289-300.

[19] Metzger and Platt, (1987). Vocational Rehabilitation of Drug Abusers. American psychological association.

[20] Miller, B. A., \& Downs, W. R. (1993). The impact of family violence on the use of alcohol by women. Alcohol Health and Research World, 17, 137-143. 
[21] Pitel, L., Madarasova Geckova, A., Kolarcik, P., Halama, P., Reijneveld, S. A., \& van Dijk, J. P. (2012). Gender differences in the relationship between religiosity and health-related behavior among adolescents. Journal of Epidemiology and Community Health, 66 (12), 1122-1128.

[22] Rehm, J., Mathers, C., Popova, S., Thavorncharoensap, M., Teerawattananon, Y., \& Patra, J. (2009). Global burden of disease and injury and economic cost attributable to alcohol use and alcohol-use disorders. Lancet, 373, 2223-33.

[23] Saunders, S. M., Resnick, M. D. Hoberman, H. M. \& Blum, R. W. (1994) Formal Help-Seeking Behaviour of Adolescents Identifying Themselves as Having Mental Health Problems"e, Journal of American Academy Child and Adolescent Psychiatry, 33 (5), pp. 718-728. 